\title{
Barriers to accessing data are bad medicine
}

$\mathrm{C}$ anada needs to "get its act together" so researchers can benefit from national investments in electronic health records, says researcher Dr. Robyn Tamblyn of McGill University in Montréal. "Each province has population-wide information on hospitalizations, long-term care, physician visits, lab results and diagnostic imaging, and some information on drugs dispensed. Only a couple of countries in the world have this. We could benefit from the richness of all of this" to shed light on clinical care and health services, says Tamblyn, who works in McGill's Department of Epidemiology, Biostatistics and Occupational Health.

Tamblyn is one of many researchers in Canada frustrated by overwhelming barriers to using health care data in research. They point to disconnected databases and concerns about privacy of personal health information as the main reasons data on everything from diagnoses to prescriptions remain locked down. They say other countries have figured out ways to use the data in research, but Canada lags.

There are exceptions. This year, a study by Tamblyn showed that more than $30 \%$ of prescriptions given to primary care patients in Quebec were not filled (Ann Intern Med 2014;160:44150). The findings came from merging data from provincial health insurance and drug insurance databases.

Ontario is likewise "lucky" to have access to databases from the Institute for Clinical Evaluative Sciences (ICES), says Tara Gomes, the principal investigator at the Ontario Drug Policy Research Network.

ICES researchers can follow a patient's path through the health care system by using the patient's health insurance number. This couldn't happen in many provinces, where health care numbers and other personal information are restricted under privacy laws.

Provincial laws differ and are interpreted differently, Tamblyn says. In some provinces, managers evaluate research-

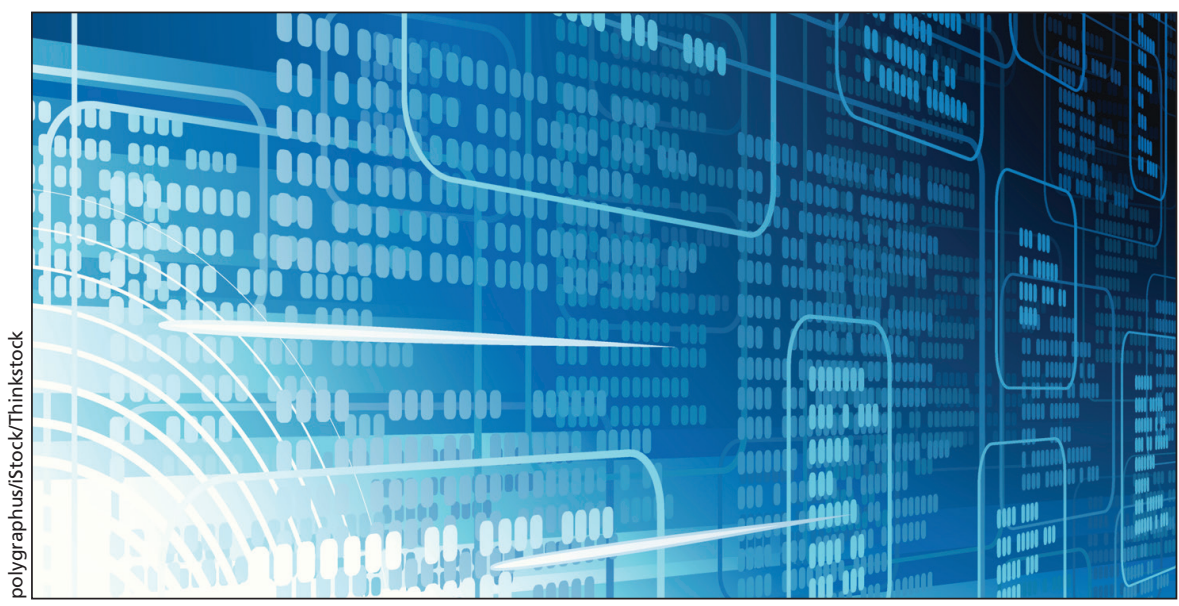

The lack of access to data makes it challenging to do interprovincial research.

ers' requests for information. "It's easier to say no than it is to say yes."

Even in provinces with organizations like ICES, it may not be possible to share data across provincial borders. This hit home recently when Gomes wanted to run analyses outside of Ontario, linking drug information to diagnosis information. There were privacy concerns.

"It introduces challenges to doing interprovincial research," she says. Australia and New Zealand often compare drug data across their country, but this is usually not possible in Canada.

Bringing data together from multiple sources allows for comparative research and increasing sample sizes. But Dany Doiron, research coordinator with Maelstrom Research at the Research Institute of the McGill University Health Centre, says there are barriers to pooling data, such as the lack of provincial resources to accommodate researchers' requests for data and varied formats.

There are initiatives underway to solve some of these problems. The Office of the Information and Privacy Commissioner advocates an approach called "privacy by design," in which privacy protection is built into the way data are collected, stored and used.

One of the main methods for protecting privacy is "deidentification" of data. Ontario's Personal Health Information
Protection Act (2004) exempts deidentified health care data from protection and allows organizations such as ICES to get data from health care providers, then deidentify and analyze them.

Manitoba and British Columbia have had organizations similar to ICES for many years. Recently, several other organizations have started to use health care data for research, including a health analytics network in Alberta and a population health data organization in Nova Scotia.

Two of the largest national cohort studies in Canadian history - the Canadian Longitudinal Study on Aging and the Canadian Partnership for Tomorrow Project - are solving the privacy problem by asking participants for broad consent in advance to use and link their data. They plan to collect data for about 25 years and link it to provincial health databases on hospitalizations, physician billing and prescription drugs. One objective is to make data available to many investigators across Canada for a number of different research questions not yet imagined, says Doiron.

Gomes hopes Ontario's infrastructure will be adopted across Canada so people can "answer important health research questions." - Carolyn Brown, Ottawa, Ont.

CMAJ 2014. DOI:10.1503/cmaj.109-4894 